\title{
Modeling IN-based service control capabilities as part of TMN-based service management
}

\author{
T. Magedanz \\ Technical University of Berlin \\ Open Communication Systems, Hardenbergplatz 2, 10623 Berlin, Germany \\ Phone: +49-30-25499229, Fax: +49-30-25499202
}

\begin{abstract}
IN and TMN standards represent the key constituents of future telecommunication environments. Since both concepts have been developed independently, functional and architectural overlaps exist. The harmonization and integration of IN and TMN is therefore currently in the focus of several international research activities. This paper investigates the thesis as to whether IN service features may be substituted by corresponding TMN management service capabilities. This means that service control of telecommunications services could be regarded as being part of the functional scope of TMN service management. Therefore this paper analyses the relationship between IN service control and TMN service management and examines, if and how TMN concepts with respect to functional and architectural aspects could be used as a basis for the provision of IN-like service capabilities for a variety of communication applications in a unified way.
\end{abstract}

\section{Keywords}

Customer Profile Management, IN, Service Control, Service Management, TMN

\section{INTRODUCTION}

In the light of a broad spectrum of different bearer network technologies (i.e. PSTN, ISDN, BISDN) the service-oriented network architecture of the Intelligent Network (IN) concept is intended to unify the creation, provision and control of advanced telecommunication services above these heterogeneous networks in a highly service-independent manner. Hence, it can be considered as the basic "network" architecture for the realization of sophisticated telecommunication services in the coming age. The Telecommunications Management Network $(T M N)$ provides the world-wide accepted ultimate framework for the unified management of all 
types of telecommunication services and the underlying networks in the future. It provides the basis for the modeling of management services, management information and related management interfaces. Both concepts were standardized at the beginning of the 1990's within the international standards bodies [Q.12xx], [M.3010].

IN and TMN are closely related in the future telecommunications environment, since they cover complementary aspects, i.e. service creation, provisioning and management [Maged-93a]. Nevertheless, both concepts are not harmonized with respect to functionality, architecture and methodologies. Consequently a harmonization and integration of both concepts is strongly required for the target telecommunication environment and therefore subject of several international research activities and the standards bodies. Generally two evolutionary steps can be identified for that integration:

1. The application of TMN concepts for the management of IN services and networks in the medium term time frame, since the first set of IN standards has not addressed this issue.

2. The long term integration of $\mathbb{I N}$ and $T M N$ within a common platform allowing the integrated creation, provision and management of future telecommunication services, comprising both communication and related management capabilities represents the ultimate target scenario.

This paper is related to the long term IN/TMN integration and proposes a new integration approach of IN and TMN concepts, taking into account the findings of research related to the medium term TMN-based management of INs [Maged-93c]. Comparing the increasing scope of emerging TMN (service) management services with the capabilities offered by IN services it could be recognized that there is an overlap of functionality since IN service features focus on the control and management of bearer transmission services (e.g. telephony). The reason for this functional overlap between IN and TMN stems from the fact that most of the IN service features were designed many years ago, when standardized (service) management concepts were not available, while facing market needs for enhanced "bearer" service capabilities and emerging customer control requirements. Consequently the $\mathrm{IN}$ could be regarded as a short term realization of a "service management network".

In contrast to existing approaches for the long term integration of IN and TMN [NA-43308], this paper proposes a different evolution scenario from current IN environments towards a long term telecommunications environment taking into account the increasing significance of Open Distributed Processing (ODP) standards and emerging results of the TINA Consortium. Therefore this paper studies the relationship between IN service control and TMN service management in more detail and investigates if and how TMN concepts could be used for the provision of IN-like service control capabilities.

The basic idea for this approach is to model (IN) service data, i.e. the "customer profile" located in the Specialized Data Function $(S D F)$, as management information in a service related Management Information Base (MIB) and to provide access to this information via standardized management protocols. This means that IN service logic programs will be substituted by TMN management services, which requires a replacement of the IN Service Control Function (SCF) by a TMN Operations System Function (OSF). The advantage of this idea is that no distinction has to be made between service control and service management, since future TMN systems could provide also IN-like service control ("call management") capabilities in a uniform way to a variety of future telecommunication services.

Therefore the following section examines the relationship between IN service control and TMN service management in more detail. Section 3 provides a brief comparison of IN and TMN functional capabilities. Section 4 provides a possible mapping of IN and TMN architectures, indicating how TMN functional elements could be used to provide IN-like management capabilities for arbitrary bearer services. An example for a TMN-based realization of the Time Dependent Routing (TDR) service features will illustrate the adopted approach in section 5. Section 6 outlines the future perspectives. A short summary concludes this paper. 


\section{IN SERVICE CONTROL VERSUS TMN SERVICE MANAGEMENT}

The historical separation of service control (SC) and service management (SM) has to be reviewed in the light of enhanced customer control capabilities offered by advanced telecommunication services and the enlarging scope of TMN management systems. Unfortunately there exists no unique definition of the relationship between service control and service management in the literature and a fuzzy borderline exists. Nevertheless, we try to illustrate the historical difference.

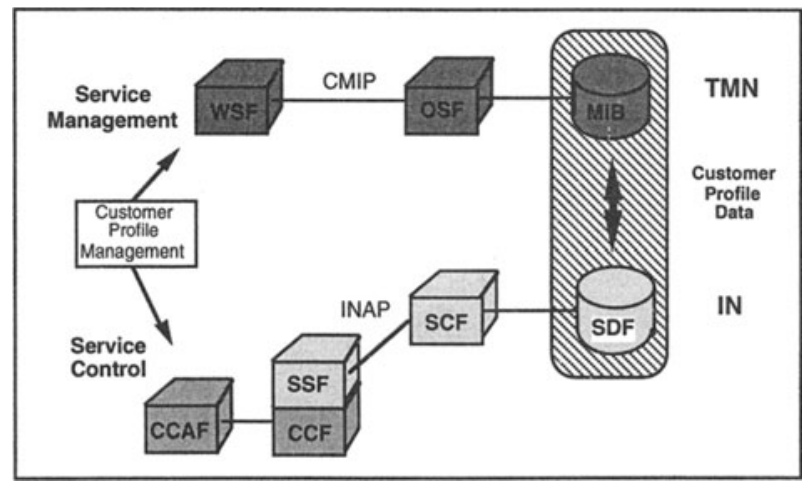

Figure 1 Customer Profile Management at the borderline between SC and SM.

Typically the term service control will be used for the real-time interpretation of (customerspecific) service data during service execution and for the manipulation of that service data for a specific customer. IN is regarded as a typical concept for the provision of service control capabilities in a network-independent way. A centralized Service Control Function (SCF), hosting the IN service logic program, interacts with a Specialized Data Function (SDF), which hosts in accordance with the subscribed service features of an IN service the customer-specific data in a "Customer Profile". Interactions between the switches, i.e. a Service Switching Function (SSF), and the SCF, which are required for IN call processing, are realized via the signalling network, i.e. the IN Application Protocol (INAP) [INAP-93]. A particular service feature, Customer Profile Management (CPM), allows customers to perform limited modifications on their service parameters in the customer profile, e.g. for rearrangement of call forwarding numbers. This access will be realized like a normal IN call via the signalling network, as indicated in Figure 1 (lower part).

On the other hand service management means administering a service on a global base, i.e. for all customers by a service provider or service subscriber mostly without any real-time constraints. TMN represents the world-wide accepted concept for the provision of service management capabilities. The TMN management services are hosted by an Operations System Function (OSF) which accesses a corresponding Management Information Base (MIB) hosting the required management information, modeled as Managed Objects. Access to the OSF from a Workstation Function (WSF) is realized via OSI's Common Management Information Protocol (CMIP) [ISO-9596-1]. Typical areas of service management are Accounting Management (e.g. billing) and Performance Management (e.g. QoS), but also Configuration Management, comprising service installation and reconfiguration as well as customer administration.

In addition RACE TMN projects [H400] have defined a new management functional area, referred to as Customer Query \& Control $(C Q \& C)$, which allows customers to read and/or 
modify specific (service) management information via a WSF. This means that besides the installation and modification of IN service triggers and IN service logic programs, etc. access to the customer profile data is also subject to TMN service management. This is also depicted in Figure 1 (upper part). But this requires modeling the customer data also as management information in a "Customer Management Profile".

For the following considerations it has to be stressed that there are two access types to the customer profile data:

- service management access, i.e. the initialization, customization and manipulation of the customer profile data by the customer or service provider. This access has only limited realtime constraints although some modifications, e.g. an user registration update, should go into effect immediately. This is one major attribute of IN services.

- service control access, i.e. the interpretation of the customer profile data during the service execution of an IN service for "controlling" a (bearer) service. This access is required by the SCF for service (feature) execution and is subject to real-time constraints.

Taking recent TMN-based IN management approaches into account, there is the general trend towards duplication of the customer service data in two separate profiles; one "customer management profile" within the TMN system supporting service management access and a corresponding IN "customer profile" in the SDF for service control access. This approach necessitates a mapping of data modifications initiated in each of both profiles. Based on the assumption that IN services can be regarded as specific (bearer) connection management services (see next section for more details), it seems sensible to use the customer management profile also for the service control access! In addition, it has to be studied, if the SCF could be modelled as a specific (real time) OSF, where IN services will be modelled as specific TMN management services. This will be addressed in the following two sections.

\section{MAPPING OF IN SERVICES TO TMN MANAGEMENT SERVICES}

Based on the previous considerations, in particular the usage of the customer management profile data for service execution access, it is necessary to investigate the functional relationships between IN services and TMN service management services in more detail. A mapping of IN services and service features [Q.1211] to corresponding TMN management services [M.3020], [M.3200] requires a careful analysis of IN services and service features with regard to their management functionality. Such an analysis has been performed within the BERKOM II project "IN/TMN Integration" [BERKOM-94a].

This analysis was primarily concerned with the assignment of the IN services and service features to the Telecommunication Management Functional Areas (TMFAs) [CFS-H400]. The TMFAs have been defined for structuring the management requirements in telecommunications networks. Thus, they provide a means for defining appropriate TMN management services. The management functionality has been determined by the criterion whether an $\mathrm{IN}$ service or service feature has management related tasks that can be assigned to one or more of the ten identified TMFAs as depicted in Figure 2.

The motivation for not mapping the IN services and service features directly to the TMN management services is to gain a management functionality analysis that is universal and independent of the management functionality of specific TMN management services. In addition, it has to be stressed that today only a few management services have been defined for specific application areas. The analysis leaves the common management requirements of IN services and service features out of consideration. For example, service accounting requires functions for collecting and processing service accounting information to generate bills for service usage. 
The analysis has revealed that a lot of the IN services (features) contain complex management functionalities. For most of the service features an assignment to the TMFAs could be made. However, a one to one mapping, i.e. the assignment of the management functionality of an IN service or service feature onto one TMFA, could seldom be found. Mostly, the management functionality is assigned to more than one TMFA leading to the assumption that an IN service or service feature can only be replaced by more than one TMN management service. Examining the IN services and service features closely it is striking that a lack of exact description and specification makes the analysis of the management functionality very difficult. Nevertheless, through the analysis of the individual IN service and service feature, the inherent management functionality could be clarified and determined.

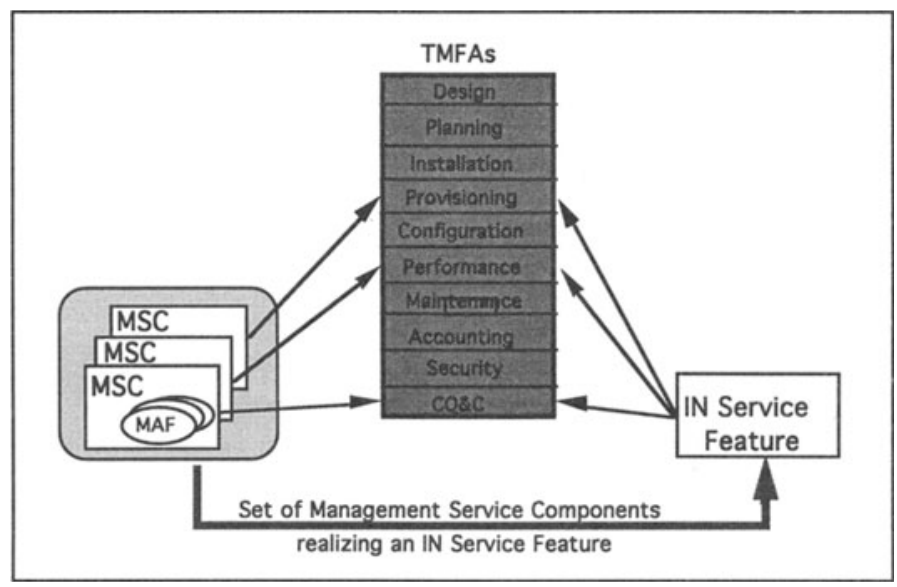

Figure 2 Mapping of IN services to TMN management services based on TMFAs.

In general, a set of management service components is needed to realize the functionality of an IN service feature. For most of the examined IN services and service features TMN management services and management service components or functions, respectively, could be found providing the management functionality of the original. After comparing the IN service features with the enlarging scope of TMN management services (MSs) the following mapping of service features (SFs) to corresponding management services can be derived:

- Customer $Q \& C$ management MSs could be used for general modifications of subscriber profiles, replacing the Customer Profile Management SF.

- Configuration management MSs could be used for realizing flexible network access and routing procedures, replacing Private Numbering Plan, Origin/Time-Dependent Routing, One Number, Call Distribution SFs, etc.

- Accounting management MSs could be used for flexible accounting procedures, replacing Premium Charging, Split Charging and Reverse Charging SFs.

- Security management MSs could be used for flexible screening options, replacing Closed User Group, O-/T-Call Screening, Authentication and Authorization Code SFs.

- Performance management MSs could be used for the provision of customer specific service statistics replacing the Call Logging and Statistics SFs. 


\section{MAPPING OF IN AND TMN ARCHITECTURES}

Based on the previous considerations it becomes clear that IN services features provide many (service) management related capabilities and could be replaced by TMN management services from a functional perspective. This section addresses the architectural issues for a TMN-based provision of IN-like service features. Taking into account the considerations of the introduction, basically two major steps can be distinguished.

\subsection{TMN-Based Service Management of INs}

The first step represents the application of TMN concepts for IN service management. Within this approach the IN functional entities Service Management Agent Function (SMAF) and Service Management Function $(S M F)$ will be replaced by the TMN functional entities Workstation Function (WSF) and Operations System Function $(O S F)$ for Service Management, respectively. The service execution related IN functional entities, namely SSF, SCF, SDF, and SRF will have to be modeled as TMN Network Element Functions (NEFs) in order to manage the service related data on these elements, e.g. service triggers, service logic programs, service data, etc. This is illustrated in Figure 3.

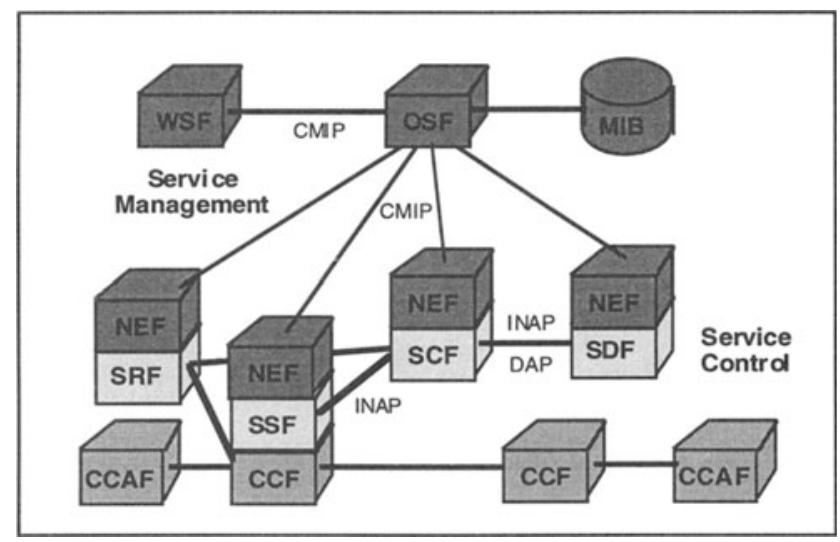

Figure 3 TMN-based service management of IN.

In addition, IN Customer Profile Management (CPM) capabilities, originally modeled as IN service feature, will have to be realized by TMN Customer Query \& Control $(C Q \& C)$ management services (CMIS versus INAP). This is due to the fact that most IN service features require complex data manipulation capabilities, e.g. Time Dependent Routing (TDR) table initialization, which could not be realized in an efficient way via the IN CPM service feature from a simple telephone set. This means that there will be a "Customer Management Profile" in a corresponding Management Information Base (MIB) either co-located with the SDF or within the corresponding OSF-S. The development of specifications for the TMN management of IN is currently within the focus of several research projects within EURESCOM and RACE. In addition, the IN standardization related to IN CS-2 is also investigating this important topic. Interested readers are referred to [Maged-93b] for more information on this subject. 


\subsection{TMN-based (IN) Service Control}

Based on the considerations of section 3 and the fact, that most of the data required by the IN service features will be modeled in addition as management information in order to be managed by TMN management service, e.g. CQ\&C services, it seems to be straight forward to make use of this management data for service control support. This means that there should be an integration of service control and service management approaches and concepts. It has to be stressed that there exists no one-to-one mapping of IN service execution related functional elements and TMN functional elements, due to the conceptual differences (IN function orientation versus TMN object orientation). Nevertheless, specific IN functional elements could be replaced step by step through TMN elements.

The first IN functional element to be replaced is the SDF. Based on the considerations of section 2 it seems likely, that the IN SDF will become a TMN MIB, containing the customer management profile. This means that there will be only one customer profile which could be used for both service management and service control access, as depicted in Figure 4. The profile data will by accessed via an appropriate management protocol, i.e. the Common Management Information Protocol (CMIP) [ISO-9596-1], for both service management and service control access. Hence the SCF will have to access that data via CMIP instead of the IN Application Protocol (INAP) [INAP-93] or the Directory Access Protocol (DAP) [X.500]. The prerequisite for this approach is the availability of fast CMIP and MIB implementations. One possible solution may be to implement CMIP on top of the signalling network (i.e. on top of TCAP).

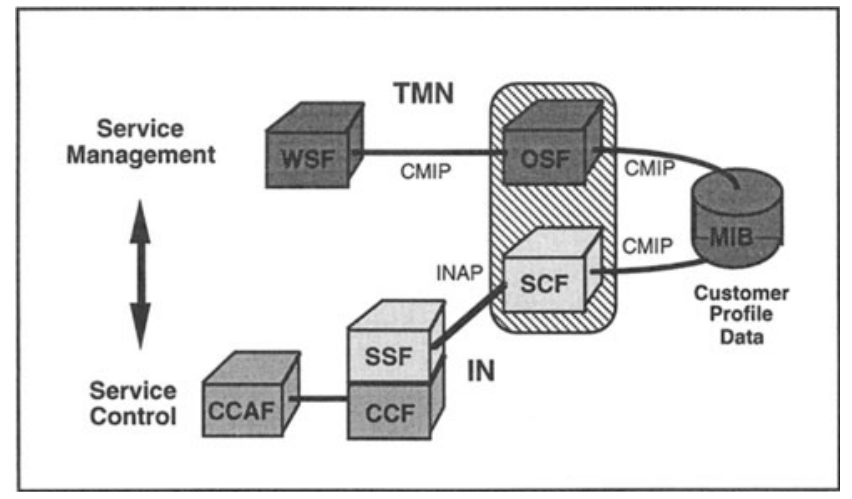

Figure 4 Common customer profile for service management and service control.

In order to realize IN services (i.e. service logic programs) by means of TMN service management services, the traditional IN SCF has to be replaced by a TMN OSF, which will run the corresponding TMN-based service control applications, as illustrated in Figure 5. This means that IN service control capabilities will be realized by appropriate TMN management services (including MSCs, MAFs, FEs) and corresponding MOs. This step represents the ultimate evolution step from the function-oriented IN environment towards a long term, objectoriented telecommunications world, such as postulationed by the emerging TINA-C initiative. It has to be stressed, that the notion of the term "OSF" in this context is a little provocative, but it is used to stress the basic idea of this evolutionary approach, namely to use the same (service management) concepts for both service management and service control. This approach is totally 
in line with the TINA-C approach of using management concepts for both management applications (such as TMN services) and telecommunications applications (such as IN services) [Pavón-94].

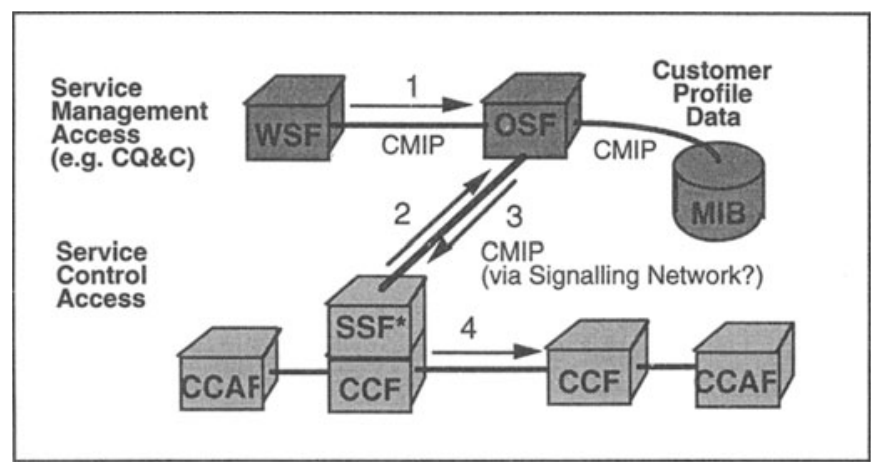

Figure 5 Target scenario for TMN-based service control.

However, there will probably no single OSF-S for running both service management and service control applications in reality. When realizing this approach it seems most likely, that there will be separate "Managers" or "Agents" for service management and service control in order to cope with the real time constraints of service control, as depicted in Figure 6. Therefore the author proposes a dedicated Service Control Agent (SCA) that will run the appropriate TMNbased service control applications, whereas a Service Management Agent (SMA) will run the corresponding service management services. Both agents will use the common customer profile located in the MIB. A similar approach for B-ISDN service control can be found in [Fukada-94].

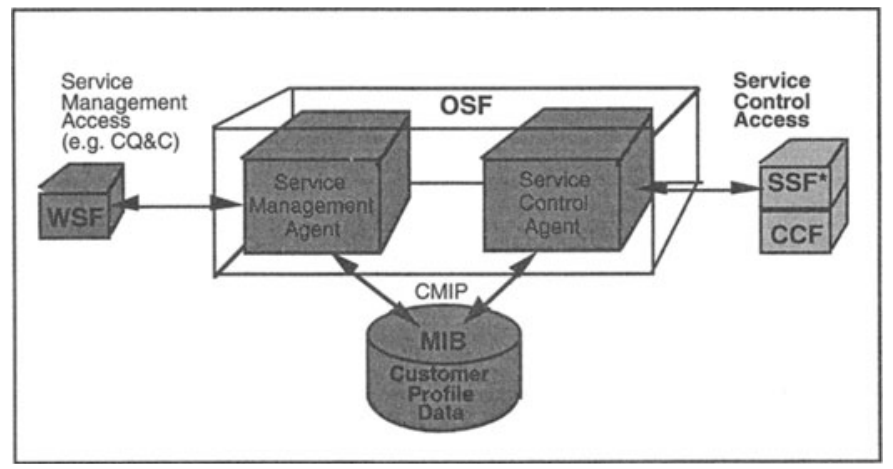

Figure 6 TMN-based service control agent and service management agent.

It has to be stressed that both "agents" will act in both "manager" and "agent" roles according to the OSI "manager-agent" paradigm; the term "agent" has only been selected in analogy to system components within an OSI environment, such as a "Directory System Agent". In TINA-C these 
components would probably referred to as "managers", such as "Session Manager" or "Service Manager" [Gatti-84].

The most challenging aspect of this scenario is the communication between traditional (IN-based) switch architectures (i.e. the SSF) and the new (TMN-based) SCA. In order to make use of CMIP instead of INAP between SSF and SCA it is necessary to introduce a new component in the SSF. Therefore the SSF is now called SSF*. This component is called Basic Call Agent (BCA), which has to recognize (based on an adapted "call model") that additional service control is required by the SCA. This is indicated in Figure 7.

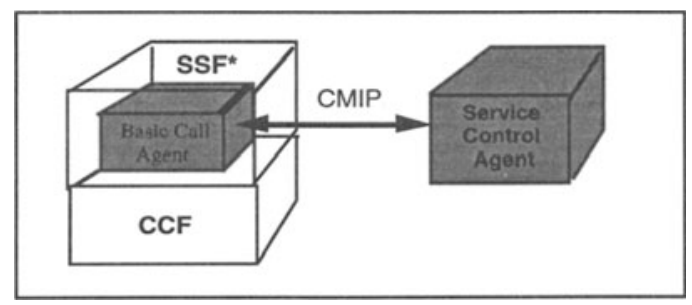

Figure 7 TMN-based basic call agent required in SSF*.

In order to support the real time requirements for service control CMIP may be implemented on top of CCS7. It has to be noted that in advanced service scenarios, such as multimedia conferencing services, much more complex call/connection models are required. The development of the SSF* and the related BCA, as well as the definition of appropriate Interworking Functions (IWFs) for supporting access to the SCA from standard switch architectures are areas for further studies.

Note that this issue represents also the basic evolution problem from current IN architectures towards TINA, since the rudimentary IN call model is not applicable to complex multimedia service sessions, and hence requires major enhancements in the switches (see [Brown-94], [Gatti-94]). Therefore, TINA has defined a corresponding "Communication Session Manager" which makes use of a "Connection Manager" in order to cope with the emerging separation of connection and call (in multimedia context replaced by the more appropriate term "session") control.

\section{A REALIZATION SCENARIO}

It is the purpose of this section to demonstrate how IN service features and thus IN services could be realized by TMN concepts. Therefore the Time-dependent Routing (TDR) service feature has been chosen as an example.

\subsection{TMN-based TDR Service Management}

The TDR service feature is representative of all service features that act on "table" Managed Objects (MOs). The operations that are performed on table MOs are almost identical. The "TDR" MO, representing a subclass of the table MO which is contained in a "Service" MO within the customer profile, has first to be created and initiated by the "Create/Delete Table" and "Set Table" Functional Elements (FEs). Before the customer is allowed to access the TDR MO (e.g. for 
adding a table entry), security functions check his/her identification and authorization. Then the customer can access the TDR table MO for modifying it (see arrow 1 in Figure 5 in section 4.2).

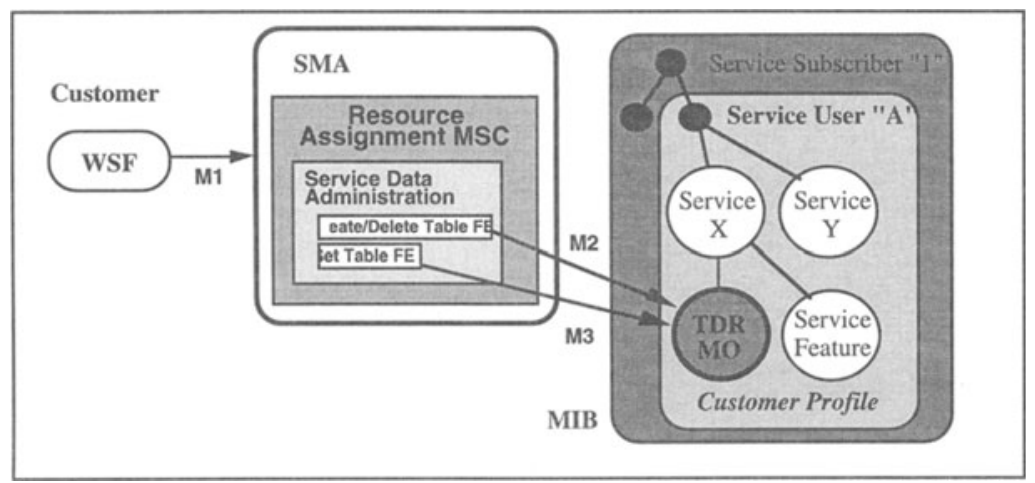

Figure 8 TMN-based service management (CQ\&C) for the TDR service feature.

Since the TDR service feature is only one component of an IN service (e.g. UPT), the provisioning of TDR will mostly take place during service provisioning unless the customer decides to add this feature to a service he/she is already provided with. The customer requests by means of appropriate CQ\&C management services via a WSF the service provider's OSF, namely the Service Management Agent (SMA), to provide the TDR service feature (M1). The procedure for TDR provisioning is depicted in Figure 8. A "Resource Assignment" Management Service Component (MSC) is addressed for the provisioning of service resources. The "Create/Delete Table" FE, as component of the "Service Data Administration" Management Application Function (MAF), initiates an instantiation of the TDR (Table) MO within the MIB (M2). If this operation is successful the TDR MO is initialized by the "Set Table" FE. In addition, the customer could modify and (de)activate specific entries of the TDR MO by corresponding MSCs, which reuses the "Set Table" FE.

\subsection{TMN-based TDR Service Control}

The TMN-based service control access for TDR looks similar to this scenario, with the "WSF" entity in Figure 8 replaced by a SSF*, the "SMA" replaced by a Service Control Agent (SCA). In addition, different MSCs, MAFs and FEs will be used. The following information flows could be identified in this scenario. The SSF*, namely the Basic Call Agent (BCA), will recognize during call set-up (based on the dialled number) the need for external service control support and hence will request by means of an appropriate MSC (e.g. "Find Call Destination") via CMIP support from the SCA. This means that the BCA has to act in a "manager" role in order to contact the SCA.

The SCA determines by corresponding service control MSCs, which service for which user is requested and identifies the corresponding User MO and the corresponding Service MO (by interpreting the dialled number). The Service MO itself (by an appropriate MO action) or a corresponding MAF will then check which service features, such as TDR, are activated by the customer within the customer profile, and finally determines the appropriate destination number by requesting the TDR MO for appropriate routing information. The result will be passed back to the BCA. The information flows $(2+3)$ in Figure 5 indicate how the SSF* will obtain the required information, where the OSF embodies both SMA and SCA. 
It has to be stressed that the relationship between management services and their components, i.e. MSCs and MAFs, and managed objects is a subject of ongoing research. The consequent application of a fully object-oriented approach for the MOs will probably eliminate the MSCs and MAFs to a large extend, since most of these functionalities will be embodied in future MOs by corresponding MO operations, i.e. actions. This means that the service control applications will be moved to the MOs themselves.

\section{FUTURE PERSPECTIVES}

The approach presented has been adopted within the BERKOM-II Project "IN/TMN Integration" undertaken by the Technical University of Berlin for the Deutsche Telekom Berkom (De•Te•Berkom). The objective of this project is the development of a TMN-based Personal Communication Support System (PCSS). This PCSS is based on an enhanced TMN platform, being part of the "Y" Platform [Zeletin-93] and offers IN-like service control capabilities, such as user registration and call handling procedures, supporting personal mobility and service personalization for an open set of (multi-media) communication services in a distributed office environment. All customer related data (e.g. user location, call handling data, etc.) will be stored in generic user service profiles, modeled as management data in an integrated X.500/X.700 system. This flexible profile integrates the data required for personal communications for all the services a user has subscribed to. Access to that profile for both customer control (i.e. profile manipulation) and communication services control (i.e. during service execution) will be realized by management services (components) via a common PCSS application programming interface. More information on the PCSS can be found in as described in [Eckardt-95] and [Berkom-94b].

\section{SUMMARY}

The IN can be regarded as the right concept for solving today's service provision requirements. But current IN concepts are limited in functionality and methodology, e.g. the function oriented nature of IN, since the trend in telecommunications is towards openness, reusability and in particular object orientation. Although the IN capability set approach allows for stepwise enhancements of IN functionalities it seems douptful, whether IN could keep the pace of evolution, in particular in the light of rapid progress in ATM deployment and multimedia service provisioning. Hence a major paradigm shift is required for IN evolution in the future.

Obviously there exist severe overlaps between IN and TMN. TMN is already based on object orientation, although the areas of management service design, creation and realization are still under development. Due to the ongoing integration of the telecommunications environment and the increasing availability of powerful management concepts, systems and services in the near future, it seems likely that IN concepts could be replaced in the long term by TMN concepts for telecommunication services provision. The basic advantage of this approach is that no separation of service control, i.e. core service capabilities, and service management has to be made, which is in line with TINA-C objectives. This has been illustrated in this paper.

\section{ACKNOWLEDGEMENTS}

The ideas presented in this paper have been developed within the BERKOM II project "IN/TMN Integration" performed at the Department for Open Communications Systems at the Technical University of Berlin for Deutsche Telekom Berkom (De•Te-Berkom). In addition the author thanks Jaqueline Aronsheim-Grotsch, who has investigated the management aspects within the IN services and service features. 


\section{REFERENCES}

[Berkom-94a] Berkom II Project "IN/TMN Integration", Deliverable 4: "Study on the TMN-based Realization of IN Capabilities", De•Te•Berkom, Germany, Berlin, June 1994

[Berkom-94b] Berkom II Project "IN/TMN Integration", Deliverable 5: "State of the Art in Personal Communications and Overview of the PCSS", De•Te•Berkom, Germany, Berlin, November 1994

[Brown-94] D.K. Brown: "Practical Issues Involved in Architectural Evolution from IN to TINA", International Conference on Intelligent Networks (ICIN), Bordeaux, France, October 1994

[CFS-H400] RACE Common Functional Specification (CFS) H400: "Telecommunications Management Functional Specification Conceptual Models: Scopes and Templates", November 1992

[Eckardt-95] T. Eckardt, T. Magedanz: "The Role of Personal Communications in Distributed Computing Environments", 2nd International Symposium on Autonomous Decentralized Systems (ISADS), Phoenix, Arizona, USA, April 25-26, 1995

[Fukada-94] K. Fukada et.al.: "Dual Agent System using Service and Management Subagents to Integrate IN and TMN", International Conference on Intelligent Networks (ICIN), Bordeaux, France, October 1994

[Gatti-94] N. Gatti: "IN and TINA-C Architecture: a Service Scenario Analysis", International Conference on Intelligent Networks (ICIN), Bordeaux, France, October 1994

[INAP-93] ETSI DE/SPS-3015: "Signalling Protocols and Switching - IN CS-1 Core Intelligent Network Apllication Protocol (INAP)", Version 08, Mai 93

[ISO-9596-1] ISO/IEC/IS 9596-1 I ITU-T Recommendation X.711: Information Processing Systems - Open System Interconnection - Common Management Information Protocol Definition (CMIP), 1991

[M.3010] ITU-T Recommendation M.3010: "Principles for a Telecommunications Management Network", Geneva, November 1991

[M.3020] ITU-T Recommendation M.3020: "TMN Interface Specification Methodology", Geneva, November 1991

[M.3200] ITU-T Recommendation M.3200: "TMN Management Services: Overview", Geneva, November 1991

[Maged-93a] T. Magedanz: "IN and TMN providing the basis for future information networking architectures", in Computer Communications, Vol.16, No. 5, May 1993

[Maged-93b] T. Magedanz et.al.: "Managing Intelligent Networks the TMN Way: IN Service versus Network Management", RACE International Conference on Intelligence in Broadband Service and Networks (IS\&N), Paris, France, November 1993

[Maged-93c] T. Magedanz: "Towards a Common Platform for Future Telecommunication and Management Services - Some Thoughts on the Relation between IN and TMN", Invited Paper at Korea Telecom International Symposium (KTIS'93), Seoul, Korea, November 1993

[NA-43308] ETSI DTR/NA-43308: "Baseline Document on the Integration of IN and TMN", Version 3, September 1992

[Pavón-94] J. Pavon et.al.: "Building New Services on TINA-C Management Architecture", International Conference on Intelligent Networks (ICIN), Bordeaux, France, October 1994

[Q.12xx] ITU-T Recommendations Q.12xx Series on Intelligent Networks, Geneva, March 1992

[Q.1211] ITU-T Recommendation Q.1211: "Introduction to Intelligent Network Capability Set 1", Geneva, March 1992

[X.500] ITU Recommendation X.500/ISO/IEC/IS 9594: Information Processing - Open Systems Interconnection - The Directory, Geneva, 1993

[Zeletin-93] R. Popescu-Zeletin et.al.: "The "Y" platform for the provision and management of telecommunication services", 4th TINA Workshop, L' Aquila, Italy, September 1993 